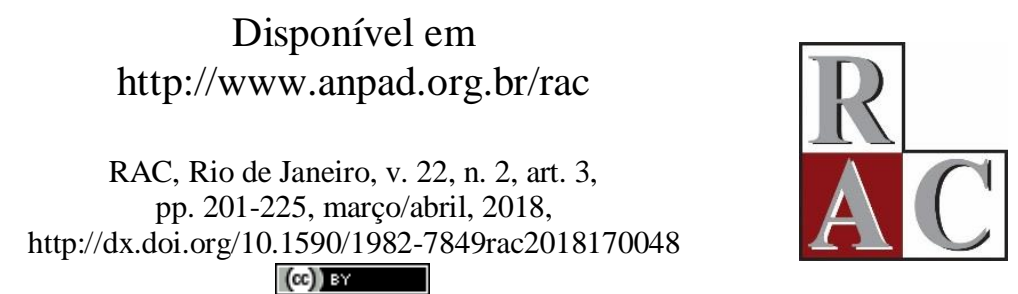

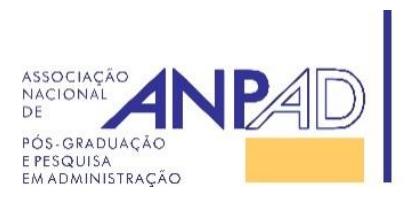

$(\mathrm{ccc}) \mathrm{EY}$

\title{
Os Papéis dos Agentes de Suporte a Empresas de Base Tecnológica
}

\author{
The Roles of Innovation Agents in New Technology-Based Firms
}

\author{
Sergio Evangelista Silva ${ }^{1}$ \\ Carlos Alberto Gonçalves ${ }^{2}$ \\ Joaquim Ramos Silva ${ }^{3}$ \\ Ana Isabel Ortega Venâncio ${ }^{3}$
}

Universidade Federal de Ouro Preto, Faculdade de Ciências Econômicas, João Monlevade, MG, Brasil ${ }^{1}$ Universidade Federal de Minas Gerais, Faculdade de Ciências Econômicas, Belo Horizonte, MG, Brasil ${ }^{2}$ Universidade de Lisboa, Lisbon School of Economics and Management, Lisboa, Portugal ${ }^{3}$ 


\title{
Resumo
}

O principal papel dos agentes de suporte à inovação (ASIs) é apoiar o desenvolvimento de empresas de base tecnológica (EBTs). Entre estes agentes destacam-se aceleradoras de empresas (AEs), incubadoras de empresas (IEs) e parques tecnológicos (PTecs). Ainda são recentes na literatura artigos que abordam estes três tipos de agentes em conjunto, o que indica que este é um tema que atualmente começa a se desenvolver, sendo, todavia, promissor em vista da importância econômica do desenvolvimento de EBTs e de ambientes de inovação. Com efeito, o objetivo deste artigo é analisar os papéis, os pontos comuns e as diferenças entre estes três tipos de ASIs, no que tange ao seu apoio ao desenvolvimento de EBTs. A abordagem utilizada é a qualitativa, em que foram obtidos dados de um total de vinte e três agentes, distribuídos entre AEs, IEs, PTecs e EBTs, localizados no Brasil, ou em Portugal. Como resultado, apresenta-se uma visão sistemática e abrangente destes três tipos de ASIs, baseada nos construtos: espaço físico e serviços básicos, gerencial, aculturação e redes de relações sociais. Adicionalmente, como implicação prática, conclui-se que este artigo pode ser utilizado como fonte de referência para o desenvolvimento, tanto de ASIs quanto de EBTs.

Palavras-chave: incubadoras de empresas; aceleradoras de empresas; parques tecnológicos; empresas de base tecnológica; agentes de suporte à inovação.

\begin{abstract}
The main role of innovation agents (IAs) is to provide support for the development of new technology-based firms (NTBFs). These agents include business accelerators (BAs), business incubators (BIs), and science parks (SPs). Studies addressing these three types of agents all together are relatively recent in the literature. This indicates that this is a new but promising topic due to the economic importance of NTBFs and innovation environments. Therefore, the purpose of this article is to examine the roles, similarities, and differences among these three types of IAs, concerning their support for the NTBFs' development. The qualitative research method was used to investigate and collect data from a sample of twenty-three agents consisting of BAs, BIs, SPs, and NTBFs, located in Brazil or in Portugal. As a result, a comprehensive and systematic view of these three types of IAs is presented based on the following constructs: physical space, basic and managerial services, acculturation, and social networks. Furthermore, as a practical implication, this article can be used as a reference source for the development of IAs and NTBFs.
\end{abstract}

Key words: business incubators; business accelerators; science parks; new technology-based firms; innovation agents. 


\section{Introdução}

A inovação tecnológica é um elemento primordial no desenvolvimento econômico e social de uma nação (Carayannis \& Zedtwitz, 2005; Dosi, 2006; Freman \& Soete, 2008; McAdam \& McAdam, 2008). Entre os vários atores do contexto de inovação, merecem destaque as empresas de base tecnológica (EBTs). Em sua maioria, elas enfrentam uma grande gama de dificuldades nas suas fases iniciais de desenvolvimento, tais como a escassez de recursos financeiros (Antolín-López, CéspedesLorente, García-de-Frutos, Martínez-del-Río, \& Pérez-Valls, 2015; Bertoni, Massimo, \& Grilli, 2011; Costa \& Torkomian, 2008; Heuven \& Groen, 2012; Lockett \& Wright, 2005), a dificuldade de acesso a estes recursos (Hueske, Endrikat, \& Guenther, 2015), uma rede fraca de relações sociais (Carayannis \& Zedtwitz, 2005; Soetanto \& Jack, 2013; Vandenbroucke, Knockaert, \& Ucbasaran, 2016) e o pouco conhecimento gerencial por parte de seus fundadores (Barbalho, Amaral, Kerbinchler, Ricther, \& Torres, 2009; Perussi \& Escrivão, 2012).

Para aumentar as chances de sobrevivência de EBTs, agentes como governos, universidades e entidades setoriais têm apoiado o desenvolvimento de ambientes cujo papel é dar suporte ao desenvolvimento destas empresas (Colombo, Cumming, \& Vismara, 2016; Colyvas et al., 2002; Franco \& Haase, 2015; Liu, Simon, Sun, \& Cao, 2011). Destacam-se, neste caso, três tipos de agentes, doravante denominados agentes de suporte à inovação (ASIs), a saber, as aceleradoras de empresas (AEs), as incubadoras de empresas (IEs) e os parques tecnológicos (PTecs) (Mian, Lamine, \& Fayolle, 2016). Em relação a estes três tipos de ASIs, a literatura sobre IEs e PTecs se encontra razoavelmente desenvolvida, enquanto são ainda recentes estudos sobre AEs (ex.: Malek, Maine, \& McCarthy, 2014; Pauwels, Clarysse, Wight, \& Hove, 2016).

Grosso modo, a literatura sobre ASIs defende que o principal papel destes agentes é dar suporte às EBTs, no que tange: (a) - à oferta de estrutura física e serviços básicos (Bollingtoft, 2012; Chan \& Lau, 2005; Iacono, Almeida, \& Nagano, 2011; J. M. Silva, Silva, \& Batista, 2012; Raupp \& Beuren, 2011; Vanderstraeten \& Matthyssens, 2012); (b) - à oferta de atividades gerenciais básicas; (c) - à aculturação (Bollingtoft, 2012; Pauwels et al., 2016); (d) - ao acesso a redes de relações sociais (Bollingtoft, 2012; Franco \& Haase, 2015; Hughes, Ireland, \& Morgan, 2007; McAdam \& McAdam, 2008; Paradkar, Knight, \& Hansen, 2015; Schwartz \& Hornych, 2008; Soetanto \& Jack, 2013; Vanderstraeten \& Matthyssensens, 2012).

Ademais, são ainda recentes estudos que realizam análises comparativas entre estes três tipos de agentes. Por exemplo, Mian, Lamine e Fayolle (2016), ao analisarem simultaneamente AEs, IEs, PTecs e centros de inovação, concluem que estes agentes devem prover infraestrutura às EBTs em termos de serviços e formação de uma rede de relações sociais, mas não abordam os pontos comuns e as especificidades destes agentes. De igual modo, embora tenha sido realizado amplo levantamento bibliográfico, não foi encontrado na literatura nenhum trabalho que analise em profundidade os papéis destes três tipos de ASIs, nem que identifique pontos comuns e peculiaridades entres eles. Adicionalmente, uma análise da literatura internacional indica que poucos textos abordam ASIs atuantes na América do Sul (ex.: Carayannis \& Zedtwitz, 2005). Não obstante, Vanderstraeten e Matthyssensens (2012) sinalizam a necessidade de aprofundamento destes estudos nesta área geográfica.

Tendo em vista esta oportunidade de contribuição à literatura, este artigo tem como objetivo analisar os papéis, os pontos comuns e as diferenças entre estes três tipos de ASIs, no que tange ao seu apoio ao desenvolvimento de EBTs. Este objetivo é orientado pela seguinte questão de pesquisa: Quais são os papéis, os pontos comuns e as diferenças existentes entre AEs, IEs e PTecs na sua função de apoio ao desenvolvimento de EBTs?

Com efeito, é apresentada uma visão comparada dos respectivos papéis com o intuito de identificar os pontos comuns e específicos de cada tipo de ASI à luz da abordagem qualitativa de caráter indutivo. A coleta de dados se baseou em entrevistas semiestruturadas, visitas e consulta a documentos obtidos de duas AEs, três IEs e cinco PEs, sendo quatro destes agentes localizados em Portugal e os restantes no Brasil, e treze EBTs localizadas no Brasil. Os principais resultados são: uma visão detalhada 
da atuação dos ASIs em quatro áreas (espaço físico e serviços básicos; gerencial; aculturação; relações sociais) utilizadas como referência; e a identificação de pontos comuns e diferenças inerentes a cada tipo de ASI nestas respectivas áreas.

\section{Referencial Teórico}

Nesta seção, primeiramente serão apresentados os três tipos de ASIs estudados (AEs; IEs; PTecs). Em seguida, serão discutidos estudos da literatura que apresentam quais são as funções destes agentes no suporte ao desenvolvimento de EBTs.

\section{Os agentes de suporte à inovação}

De modo simplificado, pode-se delimitar duas fases no desenvolvimento de uma EBT: o início do seu ciclo de vida e a maturidade (Ndonzuau, Pirnay, \& Surlemont, 2002; Perussi \& Escrivão, 2012; S. E. Silva \& Reis, 2015). A primeira normalmente é marcada por questões como: o desenvolvimento do conceito do produto, o início do processo de fabricação e comercialização e a estruturação da empresa. Na maturidade, espera-se que a EBT experimente o crescimento no volume de atividades e no faturamento, bem como a maior estruturação organizacional (Perussi \& Escrivão, 2012).

A análise da literatura sugere que são as AEs, IEs e PTecs os principais tipos de ASIs (Campanella, Peruta, \& Giudice, 2014; Chan \& Lau, 2005; Iacono et al., 2011; Pauwels et al., 2016; Ribeiro, Oliveira, \& Borini, 2012; Sofouli \& Vonortas, 2007).

Cabe às AEs e IEs dar suporte ao desenvolvimento de EBTs no início do seu ciclo de vida (Bruneel, Ratinho, Clarysse, \& Groen, 2012; M. A. O. Silva, Gomes, \& Correia, 2009; Mian et al., 2016; Pauwels et al., 2016; Rubin, Aas, \& Stead, 2015; Soetanto \& Jack, 2013). Por seu turno, os PTecs consistem em estruturas mais desenvolvidas do que estes dois primeiros tipos de agentes (Gaino \& Pamplona, 2014; Vial \& Montoro-Sanches, 2016), e seu papel é apoiar as EBTs na sua fase de maturidade (Bigliardi, Dormio, Nosella, \& Petroni, 2006). Eles têm o propósito de formar, o que é conhecido como ecossistema de inovação (Campanella et al., 2014; Hansson, Husted, \& Vestergaard, 2005; Ratinho \& Henriques, 2010; Scillitoe \& Chakrabarti, 2010), agregando EBTs e outros agentes do ambiente de inovação. No Brasil, grande parte das IEs se encontra associada a universidades, que as desenvolvem para que elas possam apoiar o desenvolvimento de EBTs criadas por pesquisadores e/ou estudantes (S. E. Silva \& Reis, 2015). Tanto as IEs quanto as AEs devem dar apoio às EBTs em questões como: cessão do espaço físico e infraestrutura, formação gerencial, auxílio na concepção do produto, formação do capital social e captação de recursos (Audretsch, Lehmann, Paleari, \& Vismara, 2016; Iacono et al., 2011; Pauwels et al., 2016; Rubin et al., 2015).

As AEs normalmente darão apoio ao desenvolvimento de uma EBT em troca de uma parcela de seu capital (Pauwels et al., 2016; S. E. Silva \& Reis, 2015), agindo, portanto, sob a expectativa de que esta empresa cresça e se desenvolva no longo prazo. Por possuírem caráter privado, as AEs normalmente desempenham um papel mais ativo no desenvolvimento de suas EBTs em questões como o aporte de recursos, a procura por investidores e o direcionamento das atividades (Malek et al., 2014; Mian et al., 2016; Pauwels et al., 2016).

\section{Os papéis dos agentes de suporte à inovação}

Carayannis e Zedtwitz (2005) destacam cinco tipos de serviços providos por IEs às suas EBTs: (a) - acesso a recursos físicos e serviços básicos; (b) - suporte de escritório; (c) - acesso a recursos financeiros; (d) - suporte ao empreendedor; e (e) - acesso a redes de contato. Estes elementos também podem ser considerados no âmbito dos outros dois tipos de ASIs contemplados nesta pesquisa (AEs e PTecs). À luz deste modelo serão consideradas quatro categorias para avaliar os serviços ofertados por 
ASIs às EBTs: (a) - a oferta de estrutura física e serviços básicos; (b) - a oferta de serviços gerenciais complementares; (c) - a aculturação; (d) - o acesso a redes de relações sociais.

Ao se pensar no apoio de ASIs às EBTs, tem-se a cessão do espaço físico, a oferta de comunicação e serviços básicos (Bollingtoft, 2012; Chan \& Lau, 2005; Iacono et al., 2011; J. M. Silva et al., 2012; Raupp \& Beuren, 2011; Vanderstraeten \& Matthyssensens, 2012). Os ASIs também podem ofertar serviços gerenciais complementares, tais como suporte contábil, jurídico, orientação gerencial e orientação de vendas, aspecto este mais presente em AEs e IEs do que em PTecs (Carayannis \& Zedtwitz, 2005; McAdam \& McAdam, 2008; Raupp \& Beuren, 2011; Ribeiro et al., 2012).

Em sua maioria, as EBTs são formadas por empreendedores cujo principal conhecimento é o técnico, proveniente da área da ciência em que atuam (Barbalho et al., 2009; S. E. Perussi \& Escrivão, 2012; S. E. Silva \& Reis, 2015). Ao instalarem suas EBTs no interior de uma AE ou IE, os empreendedores normalmente passarão pela aculturação, que envolve a participação de cursos de natureza gerencial, o desenvolvimento do plano de negócios e a incorporação de processos de gestão (Andrade, 2014; Bollingtoft, 2012; Mian et al., 2016; Rubin et al., 2015; S. E. Silva \& Reis, 2015).

Outro aspecto relevante no desenvolvimento de toda empresa é a estruturação de uma rede de relações sociais (Alvarez, Marin, \& Fonfria, 2009; Andrade, 2014), sendo este um dos principais benefícios que os ASIs podem oferecer a seus hóspedes (Mian et al., 2016; Paradkar et al., 2015; Pauwels et al., 2016; Villareal \& Calvo, 2015). As relações sociais são importantes por permitirem que as EBTs troquem informações, estabeleçam alianças, aprendam mutuamente (Caiazza, Richardson, \& Audretsch, 2015; Coradi, Heinzen, \& Boutellier, 2015; Nagano, Stefanovitz, \& Vick, 2014; Saunila \& Ukko, 2014) e tenham acesso a recursos disponíveis no ambiente externo (Caiazza et al., 2015; Cesi \& Lubatti, 2012; Paradkar et al., 2015; Rubin et al., 2015; Saunila \& Ukko, 2014).

As relações sociais das EBTs com agentes do contexto dos ASIs são classificadas em dois tipos (Soetanto \& Jack, 2013): as internas, que consistem nas relações entre as EBTs e outros agentes hospedados em um ASI, fomentadas pelo próprio compartilhamento do espaço físico (Bollingtoft, 2012; Schwartz \& Hornych, 2008; Vanderstraeten \& Matthyssensens, 2012), e as externas, que consistem nas relações com agentes do ambiente de inovação externos ao ASI. Neste sentido, a associação de uma EBT a um ASI pode ser de grande valia, à medida que ela pode se beneficiar da reputação do ASI e, assim, ter acesso facilitado a diversos agentes do mercado (Chan \& Lau, 2005; Vial \& Montoro-Sanches, 2016).

São diversos os tipos de agentes do ambiente externo ao ASI com os quais as EBTs terão algum tipo de contato, em que o ASI poderá desempenhar o papel de mediador. Entre estes agentes, destacamse universidades, agentes financeiros e mentores. A relação de EBTs com universidades se mostra relevante por causa de questões como o acesso à mão de obra, a conhecimentos técnicos específicos e a laboratórios de pesquisa (Iacono et al., 2011; Nicolaou \& Birley, 2003; Raupp \& Beuren, 2011). Todavia, esta relação dependerá das relações informais e formais previamente estabelecidas com universidades (Vial \& Montoro-Sanches, 2016). Os ASIs também podem facilitar o acesso de EBTs a agentes financeiros para a captação de investimentos (Antolín-López et al., 2015; Audretsch et al., 2016; Bertoni et al., 2011; Lindstrom \& Olofsson, 2001; McAdam \& McAdam, 2008; Raupp \& Beuren, 2011). Outro tipo de relação externa apontado na literatura é o contato de EBTs com mentores (Mian et al., 2016; Pauwels et al., 2016; Vanderstraeten \& Matthyssens, 2012), que são profissionais com ampla experiência de mercado e que podem assessorar as EBTs em questões de ordem estratégica e mercadológica.

\section{Método}

Considerando que a questão de pesquisa apresentada na introdução deste artigo é do tipo qual, os pesquisadores julgaram mais adequado adotar a abordagem qualitativa com o uso do estudo de caso para 
a condução desta pesquisa (Einsenhardt, 1989; McAdam \& McAdam, 2008; Suddaby, Bruton, \& Si, 2015; Yin, 1994). A maior adequação desta abordagem a esta pesquisa ocorre devido à natureza da questão de pesquisa; pelo fato que o entendimento dos papéis dos três tipos de ASIs estudados, seus pontos comuns e diferenças exigiam alto nível de contato do pesquisador com o contexto do estudo (Godoy, 1995, 2010); e pelo fato de os limites entre o fenômeno estudado (que consiste no papel dos ASIs como agentes de apoio a EBTs) e o contexto do estudo (qual seja, os ASIs e os agentes de seu ambiente social) não estarem claramente delimitados (Yin, 1994).

Foram realizados múltiplos casos (Yin, 1994) cujo propósito é o aprimoramento da teoria existente sobre ASIs (Einsenhardt, 1989; Shepherd \& Suddaby, 2016). Segundo Yin (1994), o uso de múltiplos casos tem como propósito a obtenção de validade externa dos resultados, para que estes possam ser passíveis de generalização analítica. Esta abordagem também permite uma visão comparativa de diferentes objetos da mesma natureza (ex.: Jaskiewicz, Heinrichs, Rau, \& Reay, 2016; McAdam \& McAdam, 2008; Robbins \& Gorman, 2015; Tenzer \& Pudelko, 2015). Com efeito, o seu uso possibilitou comparar AEs, IEs e PTecs na sua função de apoio ao desenvolvimento de EBTs, em dois contextos diferentes, Brasil e Portugal.

Os elementos conceituais identificados consistem em generalizações analíticas (Einsenhardt, 1989; Offord \& Gill, 2014; Yin, 1994), ou seja, caracterizam-se como elementos que poderão ser analisados em toda a classe de ASIs, configurando o caráter indutivo deste estudo.

A pesquisa que suscitou este artigo foi realizada segundo as etapas e o modelo conceitual apresentados a seguir. Primeiramente, foi realizada uma ampla revisão de literatura, em que se procurou identificar artigos que tratavam dos três tipos de ASIs (AEs, IEs e PTecs), objetos desta pesquisa. Para isso foram pesquisados periódicos internacionais com alto fator de impacto e que possuíam como tema principal a inovação. O mesmo procedimento foi realizado na busca de artigos em periódicos nacionais com alto nível de classificação no Sistema Qualis da Coordenação de Aperfeiçoamento de Pessoal de Nível Superior (CAPES).

A partir dos artigos, foram identificados os principais papéis de apoio dos ASIs às EBTs. Este procedimento permitiu identificar a lacuna teórica que motivou o artigo, relatada na introdução. Em face da lacuna teórica e da própria revisão da literatura, foi elaborado o roteiro de pesquisa (ver Apêndice), apresentado ao final deste artigo, sob a luz dos parâmetros: (a) - oferta de estrutura física e serviços básicos; (b) - oferta de serviços gerenciais complementares; (c) - aculturação; (d) - acesso a redes de relações sociais, baseados em Carayannis e Zedtwitz (2005). Estes quatro construtos permitiram o posicionamento dos demais elementos conceituais, identificados nos demais textos da revisão de literatura, no roteiro de pesquisa.

O formato do roteiro de pesquisa baseado nos quatro construtos apresentados acima facilitou a posterior análise comparativa dos dados dos diferentes tipos de ASIs, em que foi possível identificar pontos comuns e especificidades relativas a cada uma das classes de ASIs, bem como a cada ASI individualmente. De resto, a análise dos dados foi realizada de modo sistemático à luz de diversos textos da literatura, com vistas a verificar se eles permitiam corroborá-la, refutá-la, bem como identificar novos conhecimentos sobre o papel de ASIs no apoio a EBTs.

\section{Coleta de dados}

Foram obtidos dados de ASIs radicados no Brasil ou em Portugal, além de serem coletados dados também de EBTs, conforme descrito na introdução deste artigo e detalhados na Tabela 1. O estudo de ASIs de um lado e de EBTs do outro ocorreu no sentido de realizar a triangulação dos dados e aumentar a confiabilidade da pesquisa (Franco \& Haase, 2015; Jennings, Edwards, Jennings, \& Delbridge, 2015; McAdam \& McAdam, 2008; Vanderstraeten \& Matthyssens, 2012; Villareal \& Calvo, 2015; Tenzer \& Pudelko, 2015).

A coleta de dados em ASIs localizados no Brasil e em Portugal ocorreu pelas seguintes razões: pela predisposição de cooperação entre pesquisadores destes dois países, o que possibilitou enriquecer 
a pesquisa ao trazer uma visão a partir de diferentes pontos de vista; pela necessidade de estudos desta natureza em países em desenvolvimento, como apontado na introdução; pelo fato de que uma pesquisa realizada em dois ambientes econômicos, sociais e institucionais distintos poderia trazer resultados mais ricos e maior robustez à pesquisa.

A escolha dos agentes pesquisados ocorreu devido à facilidade de acesso, aliado ao fato de que estes deveriam oferecer dados relevantes à pesquisa. Com efeito, os pesquisadores buscaram também realizar o estudo em agentes que possuíssem representatividade dentro de sua subcategoria (AE, IE ou PTec).

O contato com os ASIs e EBTs foi feito primeiramente por meio telefônico e, posteriormente, pela troca de e-mails, com a apresentação da pesquisa e a solicitação de entrevista. Todos os agentes contatados aceitaram participar da pesquisa.

As entrevistas semiestruturadas (ex.: Tenzer \& Pudelko, 2015) foram conduzidas com o auxílio de um roteiro elaborado a partir do referencial teórico. As questões presentes no roteiro foram organizadas em torno dos construtos: estrutura física e serviços básicos; aculturação; e rede de relações sociais. O acesso a documentos de pesquisa ocorreu por meio da visita aos websites dos agentes pesquisados e pela cessão, por parte de alguns entrevistados, de documentos associados às suas atividades.

Tabela 1

ASIs e EBTs Visitados

\begin{tabular}{|c|c|c|c|c|c|c|c|c|}
\hline ASI & Tipo & Local & Entrevistados & Gên. & $\begin{array}{l}\text { Faixa } \\
\text { Etária }\end{array}$ & $\begin{array}{l}\text { Tempo na } \\
\text { atividade }\end{array}$ & Form. & País \\
\hline AE1 & Aceleradora & & Sócio-gerente & M & $20-30$ & $5-10$ & EP & Brasil \\
\hline AE2 & Aceleradora & & Sócio-gerente & M & $30-40$ & $>10$ & $\begin{array}{l}\text { Dr. } \\
\text { Farm., }\end{array}$ & Brasil \\
\hline $\begin{array}{l}\text { AE2- } \\
\text { EBT1 }\end{array}$ & EBT; software & AE2 & Sócio-gerente & M & $20-30$ & $<1$ & Gestão & Brasil \\
\hline $\begin{array}{l}\text { AE2- } \\
\text { EBT2 }\end{array}$ & EBT; software & AE2 & Sócio-gerente & F & $30-40$ & $<1$ & Gestão & Brasil \\
\hline \multirow[t]{2}{*}{$\begin{array}{l}\text { AE2- } \\
\text { EBT3 }\end{array}$} & \multirow[t]{2}{*}{ EBT; software } & \multirow[t]{2}{*}{ AE2 } & Sócio-gerente & M & $<20$ & $<1$ & $\begin{array}{c}\text { Grad. } \\
\text { EE }\end{array}$ & Brasil \\
\hline & & & Sócio-gerente & M & $20-30$ & $<1$ & $\begin{array}{l}\text { Grad. } \\
\text { EC }\end{array}$ & \\
\hline \multirow[t]{3}{*}{ IE1 } & \multirow[t]{3}{*}{ Incubadora } & \multirow[t]{3}{*}{ IE1 } & Gestora & $\mathrm{F}$ & $40-50$ & $>10$ & Gestão & \multirow[t]{3}{*}{ Brasil } \\
\hline & & & $\begin{array}{l}\text { Consultor } \\
\text { Estratégico }\end{array}$ & M & $30-40$ & $<10$ & EP & \\
\hline & & & $\begin{array}{l}\text { Consultor } \\
\text { Operacional }\end{array}$ & M & $20-30$ & $<5$ & Gestão & \\
\hline $\begin{array}{l}\text { IE1- } \\
\text { EBT1 }\end{array}$ & EBT; software & IE1 & Sócio-gerente & M & $20-30$ & $<2$ & Grad. TI & Brasil \\
\hline \multirow{2}{*}{$\begin{array}{l}\text { IE1- } \\
\text { EBT2 }\end{array}$} & \multirow[t]{2}{*}{ EBT; software } & \multirow[t]{2}{*}{ IE1 } & Sócio-gerente & M & $20-30$ & $<2$ & Grad. TI & \multirow[t]{2}{*}{ Brasil } \\
\hline & & & Sócio-gerente & M & $20-30$ & $<2$ & Grad. TI & \\
\hline
\end{tabular}


Tabela 1 (continuação)

\begin{tabular}{|c|c|c|c|c|c|c|c|c|}
\hline ASI & Tipo & Local & Entrevistados & Gên. & $\begin{array}{l}\text { Faixa } \\
\text { Etária }\end{array}$ & $\begin{array}{l}\text { Tempo na } \\
\text { atividade }\end{array}$ & Form. & País \\
\hline \multirow{2}{*}{$\begin{array}{l}\text { IE1- } \\
\text { EBT3 }\end{array}$} & \multirow[t]{2}{*}{ EBT; software } & \multirow[t]{2}{*}{ IE1 } & Sócio-gerente & M & $20-30$ & $<2$ & Gestão/TI & \multirow[t]{2}{*}{ Brasil } \\
\hline & & & Sócio-gerente & $\mathrm{F}$ & $20-30$ & $<2$ & Pedag. & \\
\hline IE2 & Incubadora & & Gestora & $\mathrm{F}$ & $30-40$ & $>5$ & Econ. & Brasil \\
\hline $\begin{array}{l}\text { IE2- } \\
\text { EBT1 }\end{array}$ & $\begin{array}{l}\text { EBT; Sensores (física } \\
\text { dos materiais) }\end{array}$ & IE2 & Sócio-gerente & $\mathrm{F}$ & $20-30$ & $<2$ & $\begin{array}{l}\text { Me. } \\
\text { Fis. }\end{array}$ & Brasil \\
\hline $\begin{array}{l}\text { IE2- } \\
\text { EBT1 }\end{array}$ & EBT; software & IE2 & Sócio-gerente & M & $20-30$ & $<2$ & $\mathrm{TI}$ & Brasil \\
\hline PTec1 & Parque Tecnológico & & Gestora & $\mathrm{F}$ & $30-40$ & $>10$ & Gestão & Brasil \\
\hline $\begin{array}{l}\text { PTec1- } \\
\text { EBT1 }\end{array}$ & EBT; software & PTec1 & Gestor & M & $30-40$ & $<2$ & $\mathrm{EE}$ & Brasil \\
\hline $\begin{array}{l}\text { PTec1- } \\
\text { EBT2 }\end{array}$ & $\begin{array}{l}\text { EBT; tecnologias } \\
\text { gerenciais }\end{array}$ & PTec1 & Sócio-gerente & M & $30-40$ & $<10$ & $\mathrm{EP}$ & Brasil \\
\hline PTec2 & Parque Tecnológico & & Gestor & M & $30-40$ & $<10$ & $\mathrm{EE}$ & Brasil \\
\hline $\begin{array}{l}\text { PTec2- } \\
\text { EBT1 }\end{array}$ & EBT; software & PTec2 & $\begin{array}{c}\text { Gestor e } \\
\text { proprietário }\end{array}$ & M & $30-40$ & $>10$ & Gestão & Brasil \\
\hline $\begin{array}{l}\text { PTec2- } \\
\text { EBT2 }\end{array}$ & EBT; software & PTec2 & $\begin{array}{c}\text { Gestor e } \\
\text { proprietário }\end{array}$ & M & $30-40$ & $>10$ & $\mathrm{TI}$ & Brasil \\
\hline PTec3 & Parque tecnológico & & Diretor & M & $30-40$ & $>10$ & $\mathrm{EE}$ & Portugal \\
\hline PTec4 & Parque tecnológico & & Diretor & M & $30-40$ & $>10$ & $\begin{array}{c}\text { Me } \\
\text { Gestão }\end{array}$ & Portugal \\
\hline PTec5 & Parque tecnológico & & Diretor & M & $50-60$ & $>10$ & Dr. EE & Portugal \\
\hline IE3 & Incubadora & PTec5 & Gestor & M & $40-50$ & $>10$ & Gestão & Portugal \\
\hline
\end{tabular}

Nota. Legenda: ASI: Agente de suporte à inovação; Aceleradora: aceleradora de empresas; Dr.: Grau de doutorado; M: Masculino; EC: Engenharia de Computação; EE: Engenharia Elétrica; EP: Engenharia de Produção; F: Feminino; Fis.: Graduação em Física; Farm.: Farmácia; Form.: Formação acadêmica; Gên.: Gênero; Gestão: Graduação na área de gestão; Grad.: Graduando; Me: Grau de Mestre; Pedag.: Graduado em Pedagogia; TI: Graduado na área de Tecnologia da Informação; Tempo na atividade: tempo em que o entrevistado desempenha a atividade.

\section{Tratamento e análise dos dados}

As entrevistas foram gravadas, com a permissão dos entrevistados, e codificadas minuto a minuto, sendo atribuídos códigos que rementem ao significado dos assuntos tratados nos respectivos períodos de gravação. Estes procedimentos se enquadram nos procedimentos conhecidos como codificação aberta e codificação axial (ex.: Jennings et al., 2015; Offord \& Gill, 2014; Tenzer \& Pudelko, 2015).

A análise dos dados foi realizada em três fases: na primeira, os pesquisadores buscaram identificar os pontos comuns entre os três tipos de ASIs, quanto ao seu papel de suporte às EBTs; na segunda, foram identificadas as particularidades de cada tipo de ASI nesta atividade; por último, foram compiladas as semelhanças e diferenças entre os diferentes tipos de ASIs. 


\section{Apresentação e Análise dos Resultados}

São apresentados, a seguir, os dados coletados nos ASIs e EBTs, seguidos de sua análise, organizados conforme os construtos: estrutura física, serviços básicos, aculturação e relações sociais.

\section{A cessão do espaço físico e o seu papel}

Na Tabela 2, são apresentados os dados básicos do espaço físico dos ASIs visitados, o seu ano de fundação, a área total aproximada disponibilizada aos ASIs e a sua localização. É possível observar que os ASIs portugueses possuem tempo de fundação maior que os ASIs brasileiros. Sobretudo os PTecs brasileiros foram fundados na presente década e estão em um nível mais recente de desenvolvimento se comparados com seus pares portugueses.

Tabela 2

Estrutura Física dos ASIs e sua Fundação

\begin{tabular}{cccc}
\hline ASI & Fundação & Área Aproximada $\left.\mathbf{( m}^{\mathbf{2}}\right)$ & Localização \\
\hline AE1 & 2010 & 300 & Brasil, Sudeste \\
\hline AE2 & 2013 & 400 & Brasil, Sudeste \\
\hline IE1 & 2003 & 400 & Brasil, Sudeste \\
\hline IE2 & 2006 & 300 & Brasil, Sudeste \\
\hline IE3 & 1992 & 300 & Portugal, Região de Lisboa \\
\hline PTec1 & 2012 & 185.000 & Brasil, Sudeste \\
\hline PTec2 & 2011 & 40.000 & Brasil, Sudeste \\
\hline PTec3 & 1991 & 12.000 & Portugal, Região de Lisboa \\
\hline PTec4 & 1995 & 3.100 & Portugal, Região de Lisboa \\
\hline PTec5 & 1992 & 150.000 & Portugal, Região de Lisboa \\
\hline
\end{tabular}

É também possível notar que as áreas destinadas aos PTecs são bem superiores às áreas destinadas a AEs e IEs. Todavia, com exceção do PTec 4, que ocupa um prédio único com área de $3.100 \mathrm{~m}^{2}$, as áreas dos demais PTecs consistem em grandes espaços não construídos. Não obstante, a visita permitiu verificar que todos os PTecs possuem áreas construídas superiores às áreas das AEs e IEs visitadas, o que corrobora a literatura que indica um maior nível de desenvolvimento dos PTecs em relação aos outros tipos de ASIs (Gaino \& Pamplona, 2014).

A forma de ocupação dos espaços pelas EBTs nos ASIs é apresentada na Tabela 3. Foram encontradas duas situações: aquela em que o ASI dá suporte somente às EBTs instaladas em suas dependências, e aquela em que este suporte também é dado às EBTs instaladas fora de suas dependências, situação denominada associação virtual. A razão apontada para esta última abordagem é a limitação de espaço físico nos ASIs. A associação virtual permite à EBT acesso aos mesmos benefícios oferecidos às EBTs instaladas nestes agentes, conforme o relato a seguir:

"A gente criou esta figura de empresa não residente para empresas que, já que o espaço acabou, está limitado, para empresas que queiram manter este vínculo, participar dos eventos, participar destas oportunidades, obter informações ... porque o vínculo com o parque pode dar uma chancela para algumas coisas (PTec1, Brasil)".

Salvo a situação do PTec2, que está instalado em um prédio, que não foi inicialmente projetado para a finalidade de abrigá-lo, todos os outros PTecs foram concebidos por meio de um projeto 
arquitetônico, contemplando espaços de convivência, para abrigar EBTs e ambientes de convenções. Esta situação denota que os PTecs, em sua maior parte, apresentam espaços mais adequados à instalação de EBTs do que as AEs e IEs, uma vez que estes últimos são abrigados em prédios adaptados e não previamente criados para a sua atividade.

No que tange ao espaço específico utilizado pelas EBTs, verificou-se três situações. Em duas IEs, foi encontrado o uso compartilhado de espaço, em que duas EBTs ocupam uma mesma sala. A segunda abordagem foi o uso do espaço comum, que foi encontrado nas AEs. Neste caso, todas as EBTs utilizam o mesmo espaço, não havendo divisórias entre elas. Por fim, em uma IE e nos PTecs, cada EBT ocupa uma sala individualmente. A maior flexibilidade no uso do espaço físico e o menor espaço ocupado por EBTs hospedadas em AEs e IEs, se comparados com seus pares hospedados em PTecs, resultam de seu menor desenvolvimento, o que corrobora vários textos da literatura (Antolín-López et al., 2015; Lockett \& Wright, 2005; Perussi \& Escrivão, 2012).

Tabela 3

\section{Oferta de Estrutura Física pelos ASIs}

\begin{tabular}{|c|c|c|}
\hline \multicolumn{2}{|c|}{ Infraestrutura e serviços } & \multirow{2}{*}{$\begin{array}{l}\text { Agentes de suporte a inovação } \\
\text { AE2; IE1; IE2; IE3; PTec2; PTec3; PTec5 }\end{array}$} \\
\hline Infraestrutura física & $\begin{array}{l}\text { Todas as empresas devem } \\
\text { estar instaladas no agente }\end{array}$ & \\
\hline & $\begin{array}{l}\text { As empresas não precisam } \\
\text { estar no mesmo espaço } \\
\text { físico }\end{array}$ & AE1; PTec1; PTec4 \\
\hline \multirow{3}{*}{$\begin{array}{l}\text { Habitação da EBT } \\
\text { hospedada }\end{array}$} & Individual & IE3; PTec1; PTec2; PTec3; PTec4; PTec5 \\
\hline & Compartilhado & IE1; IE2 \\
\hline & Comum & AE1; AE2 \\
\hline \multirow[t]{3}{*}{ Aspecto econômico } & Participação no capital & AE1; AE2; IE1 \\
\hline & Gratuito & IE2 \\
\hline & Aluguel & IE3; PTec1; PTec2; PTec3; PTec4; PTec5; \\
\hline
\end{tabular}

Quanto à sustentação econômica, foram verificadas três situações: na primeira, é feita a cessão gratuita do espaço físico; na segunda, o ASI não cobra aluguel pelo espaço, mas, em contrapartida, é proprietário de parte das cotas da EBT; e, na terceira, ocorre a cobrança de aluguel. Nota-se que a menor capacidade financeira das EBTs hospedadas em AEs e IEs faz com que elas procurem espaços gratuitos para se instalarem, ou a cessão de direitos de seu capital a quem lhes cede o espaço. Todavia, a forma de cessão do espaço físico para EBTs em seu ciclo inicial de desenvolvimento depende também de aspectos culturais e institucionais, uma vez que a IE3 radicada em Portugal cobra aluguel de suas EBTs.

Fazendo a comparação da estrutura física dos ASIs brasileiros com os portugueses, não foram observadas diferenças significativas entre as IEs visitadas. Quanto à estrutura física dos PTecs portugueses e brasileiros, foi observado um maior desenvolvimento da estrutura física dos primeiros, o que se deve principalmente ao fato de estes terem sido fundados anteriormente. Na Tabela 4 é apresentada uma breve análise acerca dos resultados sobre a ocupação do espaço físico pelas EBTs no contexto dos ASIs e sua relação com a literatura. 
Tabela 4

\section{Principais Aspectos Relativos ao Espaço Físico}

\begin{tabular}{|c|c|}
\hline Resultado & Relação com a literatura \\
\hline $\begin{array}{l}\text { EBTs instaladas em AEs e IEs são geralmente menos } \\
\text { desenvolvidas do que aquelas instaladas em PTecs. }\end{array}$ & $\begin{array}{l}\text { Este resultado corrobora Mian et al. (2016), Pauwels, } \\
\text { Clarysse, Wight e Hove (2016), Rubin, Aas e Stead } \\
\text { (2015), M. A. O. Silva, Gomes e Correia (2009), } \\
\text { Soetanto e Jack (2013) e Bruneel, Ratinho, Clarysse e } \\
\text { Groen (2012). }\end{array}$ \\
\hline $\begin{array}{l}\text { PTecs são estruturas mais desenvolvidas do que AEs } \\
\text { e IEs. }\end{array}$ & $\begin{array}{l}\text { Este resultado está em consonância com os achados } \\
\text { de Gaino e Pamplona (2014) e Vial e Montoro- } \\
\text { Sanches (2016). }\end{array}$ \\
\hline $\begin{array}{l}\text { O fato de as EBTs em ciclos iniciais de } \\
\text { desenvolvimento necessitarem da cessão gratuita ou } \\
\text { cederem parte de seu capital para a sua ocupação } \\
\text { indica a escassez de recursos financeiros destas } \\
\text { empresas. }\end{array}$ & $\begin{array}{l}\text { Esta conclusão se apoia nos seguintes estudos: } \\
\text { Bertoni, Massimo e Grilli, 2011; Costa e Torkomian, } \\
\text { 2008; Heuven e Groen, 2012; Lockett e Wright, 2005; } \\
\text { Antolín-López, Céspedes-Lorente, García-de-Frutos, } \\
\text { Martínez-del-Río e Pérez-Valls, 2015. }\end{array}$ \\
\hline $\begin{array}{l}\text { Além de ocuparem estruturas maiores e mais } \\
\text { desenvolvidas, todos os PTecs visitados, exceto um, } \\
\text { possuem espaços arquitetônicos projetados para a } \\
\text { finalidade de abrigar um parque tecnológico, } \\
\text { enquanto que nas AEs e IEs foram encontradas, em } \\
\text { todos os casos, estruturas adaptadas. Este achado } \\
\text { aponta que, provavelmente, o espaço físico dos PTecs } \\
\text { é mais adequado às atividades do ambiente de } \\
\text { inovação do que o espaço físico de AEs e IEs. }\end{array}$ & $\begin{array}{l}\text { Este resultado parece trazer uma contribuição à } \\
\text { literatura, uma vez que não foi encontrado resultado } \\
\text { semelhante nos artigos que foram utilizados na } \\
\text { revisão teórica deste artigo. Sua contribuição está no } \\
\text { fato de que, além de ter estruturas maiores, o espaço } \\
\text { físico de PTecs se mostra mais adequado ao } \\
\text { desenvolvimento de atividades inovadoras devido à } \\
\text { sua origem e formação. }\end{array}$ \\
\hline $\begin{array}{l}\text { Enquanto em PTecs a ocupação do espaço físico pelas } \\
\text { EBTs ocorre de forma individual, nas AEs e IEs a } \\
\text { ocupação pode ocorrer por meio de compartilhamento } \\
\text { do espaço por duas empresas, ou pela ocupação de um } \\
\text { mesmo espaço comum pelas EBTs. Estas diferenças } \\
\text { na forma de ocupação são decorrentes justamente do } \\
\text { nível de desenvolvimento das EBTs. A forma de } \\
\text { ocupação do espaço físico em AEs e IEs tende a } \\
\text { promover maior interação entre os membros de } \\
\text { diferentes EBTs do que a forma encontrada nos } \\
\text { PTecs. }\end{array}$ & $\begin{array}{l}\text { Este resultado traz como contribuição à literatura a } \\
\text { revelação, em maior nível de detalhe, das formas de } \\
\text { ocupação de espaço físico em ASIs pelas EBTs. } \\
\text { Também são apresentadas as considerações sobre as } \\
\text { implicações destas diferentes formas na interação e na } \\
\text { formação de um ambiente social para EBTs no } \\
\text { interior destes agentes. }\end{array}$ \\
\hline $\begin{array}{l}\text { O fato de AEs e IEs brasileiras não cobrarem aluguel } \\
\text { de suas EBTs, enquanto a IE portuguesa cobra, indica } \\
\text { que o ambiente institucional pode influenciar na } \\
\text { forma de apoio prestado às EBTs. }\end{array}$ & $\begin{array}{l}\text { Este resultado traz contribuição à literatura por indicar } \\
\text { que os elementos institucionais poderão exercer } \\
\text { considerável influência no apoio ao desenvolvimento } \\
\text { de EBTs. }\end{array}$ \\
\hline
\end{tabular}

\section{A oferta de serviços básicos}

Em todos os ASIs visitados, as EBTs têm acesso a serviços como telefone, internet, serviço postal, secretaria, entre outros. Uma síntese dos serviços oferecidos é apresentada na Tabela 5. Destaca-se um importante aspecto que é também a difusão da informação. Tendo em vista que IEs, AEs e PTecs são filiados a entidades do ambiente de inovação, eles recebem frequentemente informações do ambiente externo via internet, que são repassadas às EBTs hospedadas. A este respeito são apresentados os seguintes relatos:

"A partir do momento em que a empresa é cadastrada aqui, tudo que a gente recebe sobre inovação, palestra, oficina, curso é repassado [às EBTs hospedadas] (IE2, Brasil)". 
"Então o que nós fazemos é tratar internamente a informação e depois selecionamos quem é que parece ser os mais interessado[s] naquela oportunidade (PTec4, Portugal)".

Também a este respeito, o gestor entrevistado em um parque tecnológico revela a vantagem da instalação de EBTs neste parque, que a seu ver ocorre da seguinte maneira:

"Nós não temos só espaço, nós prestamos um serviço. A pessoa chega, tem interfone, tem internet, tem espaço de copa, tem impressora central. Quer dizer, a pessoa chega e está em condições de começar a trabalhar, sem precisar fazer mais contrato nenhum (PTec3, Portugal)."

No que tange ao acesso a outros serviços complementares, a IE1oferece serviço jurídico aos seus hóspedes, enquanto as duas aceleradoras oferecem serviço jurídico e contábil.

Tabela 5

\section{Oferta de Serviços Básicos pelos ASIs}

\begin{tabular}{ll}
\hline Infraestrutura e serviços & Agentes de suporte à inovação \\
\hline Internet, telefone e informações, secretaria, etc. & Todos os agentes visitados \\
\hline Acesso somente à consultoria jurídica & IE1 \\
\hline Acesso à consultoria jurídica e contábil & AE1; AE2 \\
\hline
\end{tabular}

Embora todos os ASIs ofereçam infraestrutura e serviços básicos aos seus hóspedes, nota-se que AEs e IEs, que são ASIs cujo papel é dar suporte às EBTs em ciclos iniciais de desenvolvimento, oferecem uma gama maior de serviços de apoio (Carayannis \& Zedtwitz, 2005; McAdam \& McAdam, 2008; Raupp \& Beuren, 2011; Vanderstraeten \& Matthyssens, 2012). Por seu turno, a não oferta destes serviços por algumas IEs estudadas denota sua deficiência neste aspecto. Já a ausência de serviços gerenciais de apoio em PTecs se dá pelo fato de suas EBTs já serem suficientemente desenvolvidas e fazerem uso destes serviços no mercado. Na Tabela 6 é apresentada uma análise acerca do papel dos ASIs na oferta de serviços às suas EBTs.

Tabela 6

\section{Principais Aspectos Relativos aos Serviços Ofertados pelos ASIs}

\begin{tabular}{ll}
\hline Resultado & Relação com a literatura \\
\hline $\begin{array}{l}\text { Enquanto AEs e IEs dão apoio ao desenvolvimento } \\
\text { das EBTs em seu ciclo inicial de desenvolvimento, os }\end{array}$ & $\begin{array}{l}\text { Este achado possui ampla sintonia com a literatura } \\
\text { (Bruneel } \text { et al., 2012; M. A. O. Silva } \text { et al., 2009; Mian } \\
\text { PTecs dão suporte às EBTs em um nível mais }\end{array}$ \\
$\begin{array}{l}\text { avançado de desenvolvimento. 2016; Pauwels } \text { et al., 2016; Rubin } \text { et al., 2015; } \\
\text { Soetanto \& Jack, 2013). }\end{array}$ \\
$\begin{array}{ll}\text { Nas AEs o provimento de serviço às EBTs é feito em } \\
\text { troca de uma parcela de seu capital. }\end{array}$ & $\begin{array}{l}\text { Esta situação está de acordo com estudos de Pauwels } \\
\text { et al. } \text { (2016) e S. E. Silva e Reis (2015). }\end{array}$ \\
\hline
\end{tabular}

Foi verificado um papel mais ativo das AEs do que IEs Esta situação encontra suporte em diversos autores e PTecs no desenvolvimento das EBTs. Este papel se manifesta em aspetos como maior proatividade na (Malek et al., 2014; Mian et al., 2016; Pauwels et al., 2016). busca de investidores para as EBTs e em um nível mais intenso de suporte no desenvolvimento do negócio.

Por seu turno, cabe aos PTecs apoiarem o desenvolvimento de EBTs em um estágio de desenvolvimento mais maduro.
Este resultado encontra suporte em Bigliardi, Dormio, Nosella e Petroni (2006). 


\section{Tabela 6 (continuação)}

\begin{tabular}{|c|c|}
\hline Resultado & Relação com a literatura \\
\hline $\begin{array}{l}\text { Os ASIs oferecem as seguintes categorias de serviços } \\
\text { às EBTs que hospedam: (a) - a oferta de estrutura } \\
\text { física e serviços básicos; (a) - a oferta de serviços } \\
\text { gerenciais complementares; (c) - a aculturação; (d) - o } \\
\text { acesso a redes de relações sociais. }\end{array}$ & $\begin{array}{l}\text { Este resultado corrobora Carayannis e Zedtwitz } \\
(2005) \text {, cujos construtos nortearam o desenvolvimento } \\
\text { deste artigo. }\end{array}$ \\
\hline $\begin{array}{l}\text { Todavia, o nível de suporte nestes aspectos dependerá } \\
\text { do nível de desenvolvimento individual do ASI, e da } \\
\text { categoria à qual pertence (AE, IE ou PTec), como será } \\
\text { melhor explicado a seguir. }\end{array}$ & \\
\hline $\begin{array}{l}\text { Todos os ASIs oferecem às EBTs que hospedam uma série } \\
\text { de serviços básicos, tais como infraestrutura de } \\
\text { comunicação e de instalação, não sendo observada } \\
\text { diferença neste quesito entre os tipos de ASIs pesquisados. }\end{array}$ & $\begin{array}{l}\text { Este resultado corrobora diversos estudos da literatura } \\
\text { (Bollingtoft, 2012; Chan \& Lau, 2005; Iacono et al., } \\
\text { 2011; J.M. Silva et al., 2012; Raupp \& Beuren, 2011; } \\
\text { Vanderstraeten \& Matthyssensens, 2012). }\end{array}$ \\
\hline $\begin{array}{l}\text { Foi observado que AEs e IEs oferecem uma gama } \\
\text { maior de serviços complementares às EBTs do que os } \\
\text { PTecs. }\end{array}$ & $\begin{array}{l}\text { Este resultado corrobora a literatura (Carayannis \& } \\
\text { Zedtwitz, 2005; McAdam \& McAdam, 2008; Raupp \& } \\
\text { Beuren, 2011; Ribeiro et al., 2012). }\end{array}$ \\
\hline $\begin{array}{l}\text { As AEs e IEs apoiam o desenvolvimento de EBTs nos } \\
\text { seguintes aspectos: cessão do espaço físico e } \\
\text { infraestrutura, formação gerencial, auxílio na } \\
\text { concepção do produto, formação do capital social e } \\
\text { captação de recursos. }\end{array}$ & $\begin{array}{l}\text { Este resultado encontra amplo suporte na literatura } \\
\text { (Audretsch et al., 2016; Iacono et al., 2011; Pauwels et } \\
\text { al., 2016; Rubin et al., 2015). }\end{array}$ \\
\hline $\begin{array}{l}\text { Embora AEs e IEs ofereçam, em média, mais serviços } \\
\text { complementares às EBTs que hospedam do que os } \\
\text { PTecs, foi observado que a gama de serviços ofertados } \\
\text { varia de AE e IE individualmente. Esta situação indica } \\
\text { que existem diferentes níveis de desenvolvimento } \\
\text { entre estes agentes. }\end{array}$ & $\begin{array}{l}\text { Este resultado parece trazer um aprimoramento à } \\
\text { literatura, uma vez que não foram encontrados, na } \\
\text { literatura analisada, resultados semelhantes. }\end{array}$ \\
\hline
\end{tabular}

\section{Aculturação e desenvolvimento estratégico da empresa}

Os principais aspectos relativos ao processo de aculturação de EBTs no contexto dos ASIs são apresentados na Tabela 7. Um ponto relevante na aculturação consiste na implantação do planejamento e controle das atividades na EBT. Elementos de aculturação podem ser vistos na seguinte fala de um dos entrevistados:

"Eles [os empreendedores das EBTs] sabem muito tecnicamente do produto, do serviço deles, mas não têm noção de negócios, não têm planejamento estratégico, não têm o próprio modelo de negócios .... Além de estabelecer estes limites institucionais, visão, missão, valores, estas coisas mais subjetivas para as pessoas terem um norte, a gente ajuda a gerenciar os processos internos da empresa (IE1, Brasil)."

Foi verificado que enquanto IEs e AEs normalmente interferem diretamente nas atividades gerenciais das EBTs, os PTecs normalmente apenas verificaram se a EBT mantém compromisso em desenvolver tecnologia, uma vez que este aspecto está diretamente relacionado à razão de existir dos PTecs. Com efeito, a maior intervenção de IEs e AEs corrobora a teoria que considera a maior necessidade de apoio a EBTs que estão em seu ciclo inicial de desenvolvimento e, como consequência, precisam de mais apoio dos ASIs (Bruneel et al., 2012; M. A. O. Silva et al., 2009; Mian et al., 2016; Pauwels et al., 2016; Rubin et al., 2015; Soetanto \& Jack, 2013).

Quanto ao nível de acompanhamento pelo ASI das atividades da EBT, nas AEs e em uma IE ele é feito diariamente, em duas IEs o acompanhamento é mensal e nos PTecs é feita apenas a verificação das atividades das EBTs com menor frequência, de modo a garantir que elas se mantenham como empresas que trabalham com alta tecnologia. 
Tabela 7

\section{Processo de Aculturação de EBTs nos ASIs}

\begin{tabular}{lll}
\hline Infraestrutura e serviços & Agentes de suporte à inovação \\
\hline \multirow{2}{*}{ Acompanhamento } & Intensivo & AE1; AE2; IE1 \\
\cline { 2 - 3 } & Mensal/Bimestral & IE2; IE3 \\
\cline { 2 - 3 } & Manutenção de sua base tecnológica & Todos os parques tecnológicos \\
\hline Ferramentas de Gestão & AE1; AE2; IE1 \\
\hline Suporte de Mentores & AE1; AE2; IE1 \\
\hline Desenvolvimento da visão estratégica & AE1; AE2; IE1; IE2; IE3 \\
\hline
\end{tabular}

Foi também observado o suporte de mentores nas duas AEs e em uma IE. Estes profissionais assessoram as EBTs em aspectos mercadológicos e no acesso à rede de contatos. Como consequência da maior interação das AEs e IEs com suas EBTs, foi observado que elas possuem um importante papel na formação de sua visão estratégica. Esta visão consiste na forma com que o empreendedor compreende o ramo em que atua e como pretende agir neste ramo (Mintzberg, Lampel, Quinn, \& Goshal, 2006). Na Tabela 8 são apresentados os principais aspectos relativos ao processo de aculturação das EBTs nos diversos tipos de ASIs.

Tabela 8

\section{Principais Aspectos Relativos ao Processo de Aculturação das EBTs}

\section{Resultado}

AEs e IEs interferem no desenvolvimento das EBTs no sentido de institucionalizarem uma série de atividades gerenciais e estratégicas.

\section{Relação com a literatura}

De certo modo, este resultado se alinha com a visão de diversos autores que relatam uma oferta maior de serviços gerenciais destes agentes às EBTs (Carayannis \& Zedtwitz, 2005; McAdam \& McAdam, 2008; Raupp \& Beuren, 2011; Ribeiro et al., 2012). Todavia, o presente estudo traz um novo achado, uma vez que mostra que AEs e IEs não só oferecem mais serviços do que PTecs, mas também exigem que diversas atividades sejam institucionalizadas, sob o pressuposto de que elas trarão maior eficiência para as EBTs.

Adicionalmente, foi notada uma maior intervenção de AEs do que IEs nas atividades das EBTs. Esta situação provavelmente ocorre devido à natureza privada das Este resultado corrobora estudos de Malek, Maine e McCarthy (2014), Mian et al. (2016) e Pauwels et al. (2016).

AEs e, portanto, ao fato que o desempenho financeiro das EBTs que hospedam está diretamente relacionado ao seu próprio desempenho financeiro.

AEs e IEs são importantes instrumentos de divulgação de conceitos e ferramentas gerenciais, utilizando-os como elementos de aculturação das EBTs.

Este resultado encontra amplo suporte na literatura (Andrade, 2014; Bollingtoft, 2012; Mian et al., 2016; Rubin et al., 2015; S. E. Silva \& Reis, 2015).

AEs e IEs possuem papel relevante na formação da visão estratégica das EBTs. Neste sentido, o ASI pode ser determinante no sucesso da EBT, uma vez que a influencia na tomada de decisões estratégicas.
Este resultado parece contribuir para o corpo de conhecimento existente na literatura, uma vez que faz uma relação entre os conceitos de visão estratégica e aspectos propriamente tratados no âmbito da inovação tecnológica. Adicionalmente, novos estudos poderão ser realizados para que se entenda melhor o desenvolvimento da visão estratégica no âmbito de EBTs em seu ciclo inicial de desenvolvimento. 


\section{Tabela 8 (continuação)}

\begin{tabular}{l} 
Resultado \\
\hline Foi notada a presença de diversos profissionais com \\
formação gerencial no comando de EBTs. Com efeito, \\
este achado parece apontar na direção de que muitas \\
EBTs atuais são originadas com grupos mais \\
heterogêneos e complementares de profissionais, o que \\
tende a aumentar as chances de seu sucesso.
\end{tabular}

As AEs e IEs possuem um importante papel no desenvolvimento das EBTs ao facilitarem o seu contato com tutores.

\section{Relação com a literatura}

Este resultado refuta a visão de Barbalho, Amaral, Kerbinchler, Ricther e Torres (2009), S. E. Silva e Reis (2015) e Perussi e Escrivão (2012), que apontam que as EBTs muitas vezes são originadas por profissionais que possuem apenas conhecimento técnico e científico, e não gerencial. Esta diferença entre o resultado desta pesquisa e a visão dos autores acima indica que o padrão de formação do pessoal das EBTs pode mudar ao longo do tempo.

Este resultado está em consonância com alguns estudos da literatura (Mian et al., 2016; Pauwels et al., 2016; Vanderstraeten \& Matthyssens, 2012).

\section{Acesso a redes de relações sociais}

\section{Formação de um ambiente social interno}

Os principais aspectos do papel dos ASIs como agentes de fomento à interação social de suas EBTs são sumarizados na Tabela 9. Como exemplo de processo de estímulo à formação de um ambiente social interno, tem-se o relato de um evento frequentemente realizado por um dos parques tecnológicos visitados:

“Temos as sessões de café da manhã * [tradução nossa do termo breakfast sessions], que ocorrem uma vez por mês, em que trazemos alguém de uma área qualquer, e promovemos uma manhã em que alguém vem falar e são convidados todos os outros [agentes do parque] (PTec5, Portugal)".

Ademais, foi observado que o espaço físico é predisponente natural para a interação entre as EBTs, se este permite proximidade física. Evidência desta questão pode ser vista no seguinte relato de uma das incubadoras:

“Em termos de incubadora, como as pessoas estão próximas fisicamente, há, de fato, oportunidade[s] de mercado[s] que são atingidas pela junção de duas ou mais empresas. (....) As empresas criam parcerias para levar determinado produto ao mercado (IE3, Portugal)".

Tabela 9

\section{Suporte dos ASIs à Interação Social de suas EBTs}

\begin{tabular}{|c|c|}
\hline Elementos de interação social interna & ASIs \\
\hline Espaço sem paredes & AE2 \\
\hline Proximidade física & AE1; AE2; IE1; IE2; IE3 \\
\hline Promove palestras de agentes externos & AE2; IE1; IE2; PTec1; PTec2; PTec5 \\
\hline Manifestou parcerias formais Prestação de serviços & IE2; PTec1 \\
\hline $\begin{array}{ll}\text { entre agentes } & \text { Desenvolvimento conjunto de produto }\end{array}$ & PTec2; IE3 \\
\hline
\end{tabular}

A AE2 é o ASI que possui a estrutura que mais fomenta esta interação, posto que todas as EBTs se encontram agrupadas em dois grandes espaços comuns, ou seja, sem paredes. Foi também verificada considerável proximidade física nas AEs, IEs e em dois PTecs (PTec2 e PTec4), em que as EBTs se encontram hospedadas em um mesmo piso, todavia separadas por paredes. Já no caso dos demais PTecs, 
a sua estrutura física maior faz com que as EBTs fiquem mais distantes umas das outras, o que pode dificultar a sua interação.

De modo geral, é consenso entre os entrevistados que a interação entre os membros de diferentes EBTs hospedadas em um ASI gera a troca de informações e conhecimentos. Adicionalmente, muitas EBTs buscam se instalar em ASIs justamente pela possibilidade de interagir com outras EBTs, conforme o relato do gestor de uma EBT, transcrito a seguir:

"Eu estou num parque tecnológico, eu busco um parque tecnológico acreditando que a relação entre as empresas consegue desenvolver todas elas (PTec2-EBT1, Brasil)".

A Tabela 10 apresenta a análise sucinta dos principais aspectos relativos à formação de um ambiente social interno nos ASIs.

Tabela 10

Principais Aspectos Relativos à Formação de um Ambiente Social Interno nos ASIs

\begin{tabular}{ll}
\hline Resultado & Relação com a literatura \\
\hline $\begin{array}{l}\text { Os ASIs atuam como entidades promotoras da } \\
\text { formação de um ambiente social interno entre suas } \\
\text { EBTs. }\end{array}$ & $\begin{array}{l}\text { Este resultado é corroborado por diversos autores na } \\
\text { literatura (Alvarez et al., 2009; Mian } \text { et al., 2016; } \\
\text { Paradkar, Knight, \& Hansen, 2015; Pauwels } \text { et al., 2016; } \\
\text { Villareal \& Calvo, 2015). }\end{array}$ \\
\hline $\begin{array}{l}\text { A formação deste ambiente se deve à interação das } \\
\text { EBTs devido à proximidade física, bem como pela } \\
\text { promoção de atividades no ASI que favorecem a } \\
\text { abertura de contato e a interação. }\end{array}$ & $\begin{array}{l}\text { Este resultado corrobora estudos como os de Bollingtoft } \\
\text { (2012), Schwartz e Hornych (2008) e Vanderstraeten e }\end{array}$ \\
\hline $\begin{array}{l}\text { Além do espaço físico, o desenvolvensens (2012). } \\
\text { eventos no interior dos ASIs poderá favorecer a } \\
\text { interação entre os membros das EBTs e produzir, } \\
\text { como consequência, a formação de relações sociais. }\end{array}$ & $\begin{array}{l}\text { Este resultado traz uma nova visão à literatura, mostrando } \\
\text { de modo mais detalhado como os ASIs podem } \\
\text { social interno. }\end{array}$ \\
$\begin{array}{l}\text { A existência de EBTs com capacidades } \\
\text { complementares nos ASIs pode agir como elemento } \\
\text { predisponente à formação de parcerias para suprir } \\
\text { demandas do mercado. }\end{array}$ & $\begin{array}{l}\text { Este resultado traz uma nova visão à literatura, que } \\
\text { mostra que, embora o ambiente do ASI possa predispor a } \\
\text { interação entre EBTs, esta tenderá a ser mais intensa } \\
\text { devido às suas características e seus interesses mútuos. }\end{array}$ \\
\hline
\end{tabular}

\section{Formação de um ambiente social externo}

No que tange ao papel do ASI na relação de suas EBTs com o meio externo, foram analisadas as interações com os seguintes tipos de agentes: financeiros, universidades e ambiente de inovação. As interações se encontram sumarizadas na Tabela 11. 
Tabela 11

\section{Suporte dos ASIs à Interação Social de suas EBTs com Agentes Externos}

\begin{tabular}{|c|c|c|}
\hline \multicolumn{2}{|c|}{ Elementos de interação social externa } & \multirow{2}{*}{$\begin{array}{c}\text { ASIs } \\
\text { AE2 }\end{array}$} \\
\hline Agentes & Análise sistemática de investidores (venture capital) & \\
\hline Financeiros & Intermedia relações com investidores anjo & AE1 \\
\hline & $\begin{array}{l}\text { Procura de parceria com investidores e fundos } \\
\text { públicos }\end{array}$ & PTec1; PTec2; PTec3; PTec4; PTec5; \\
\hline \multirow[t]{3}{*}{ Universidade } & Intermedia relações das EBTs & IE1; IE2; PTec2; PTec4; PTec5 \\
\hline & Relação institucional & $\begin{array}{l}\text { IE1; IE2; IE3; PTec1; PTec2; PTec4; } \\
\text { PTec5 }\end{array}$ \\
\hline & Relações com agentes específicos da universidade & AE1 e AE2 \\
\hline
\end{tabular}

A relação com agentes financeiros se mostrou mais ativa nas duas AEs visitadas em comparação com os demais ASIs. Esta situação é considerada desta maneira porque estes agentes fazem o efetivo agenciamento de investidores para as suas EBTs, o que ocorre no nível individual ( $a d$ hoc). Como pode ser visto no relato abaixo, a política da AE1 é não só procurar investidores anjo para suas EBTs, mas investidores que, além de disponibilizarem capital, tenham conhecimento e experiência no ramo de atividade das EBTs: "Trazer um investidor qualquer ou eu trazer um investidor da área é algo que coloca a pessoa que tem uma rede de contatos na área na frente daquela que não tem. E aquilo ali acaba promovendo a aceleração do negócio, faz sair mais rápido (AE1, Brasil)".

Por seu turno, nos PTecs do Brasil e de Portugal foram observados esforços de captação de recursos de agentes públicos, na forma de linhas de financiamento destinadas às EBTs instaladas nestes agentes.

A universidade se mostra um elemento presente na maioria dos ASIs pesquisados. Foram identificados três tipos de relações entre estes agentes e as universidades: (a) - a situação em que o ASI intermedia a relação entre uma EBT e algum agente da universidade, ou seja, o acesso a algum serviço, laboratório de pesquisa, ou suporte de um pesquisador; (b) - a relação institucional, em que o ASI efetivamente está incorporado ao organograma de uma universidade, ou a universidade é um agente chave no seu controle; (c) - a relação do ASI com pesquisadores da universidade, normalmente para o aprimoramento de técnicas gerenciais do ASI. Tem-se, abaixo, o relato do gestor entrevistado no PTec2, em que este parque obrigatoriamente intermedia toda relação de suas EBTs e a universidade à qual pertence:

"Eles não podem fazer direto [contato com entes da universidade]. O parque tem que intermediar [este contato] (PTec2, Brasil)".

No que se refere à relação com agentes do ambiente de inovação, é importante considerar a relação de EBTs com mentores, graças ao intermédio de seus respectivos ASIs, como pode ser visto no seguinte relato:

"Então a gente vai buscar entre os mentores quem é que tem esta permeabilidade no mercado, para facilitar a apresentação e a validação da venda. ... Este é o principal diferencial da aceleradora. Se você não tem quem te apresente, você é qualquer outro vendedor batendo à porta. Quando você tem alguém de peso, quando você tem uma [executiva amplamente conhecida] ligando para o presidente da empresa, e dizendo: - Receba, porque eu confio. É totalmente diferente (AE2, Brasil)".

Na Tabela 12 é apresentada uma análise dos principais aspectos relativos ao papel dos ASIs na formação do ambiente social externo das EBTs. 
Tabela 12

\section{Principais Aspectos Relativos À Formação De Um Ambiente Social Externo nos ASIs}

\begin{tabular}{l} 
Resultado \\
\hline Os ASIs possuem o papel de facilitadores da formação \\
de relações sociais de suas EBTs com agentes do \\
ambiente de inovação. \\
De modo geral, os ASIs dão suporte às suas EBTs no \\
que tange à relação com agentes ligados às \\
universidades. Esta interação ocorre principalmente \\
pela busca de conhecimento \\
técnico-científico, do uso de laboratórios de pesquisa e \\
pelo acesso à mão de obra com alto nível de \\
especialização.
\end{tabular}

A interação de ASIs institucionalmente relacionados à universidade é maior do que a interação de ASIs que não possuem vínculo institucional com a universidade.
Os ASIs dão apoio às suas EBTs para a captação de investimentos de agentes financeiros.

\section{Relação com a literatura}

Este resultado encontra suporte em Chan e Lau (2005)

e Vial e Montoro-Sanches (2016).

Este resultado se coaduna com alguns estudos da literatura (Iacono et al., 2011; Nicolaou \& Birley, 2003; Raupp \& Beuren, 2011).

Este resultado confirma a visão de Vial e MontoroSanches (2016).

Este resultado corrobora diversos textos da literatura (Antolín-López et al., 2015; Audretsch et al., 2016; Bertoni et al., 2011; Lindstrom \& Olofsson, 2001; McAdam \& McAdam, 2008; Raupp \& Beuren, 2011). Como conhecimento novo a respeito, tem-se que o apoio na captação de recursos ocorre em dois sentidos: pela facilitação da realização de investimentos, devido à reputação do ASI, e pelo fato de os gestores do ASI possuírem conhecimento e experiência neste processo de captação de recursos.

Foi observado um papel mais ativo das AEs em relação às IEs em intermediarem contato com agentes financeiros, o que corrobora novamente a visão de que Este resultado é também apontado por alguns estudos recentes da literatura (Malek et al., 2014; Mian et al., 2016; Pauwels et al., 2016).

AEs possuem um papel mais ativo no desenvolvimento de EBTs. Novamente, este maior dinamismo das AEs se deve ao incentivo financeiro, uma vez que o desenvolvimento das EBTs poderá lhes trazer lucro, enquanto que esta relação normalmente não é encontrada nas IEs.

O serviço de suporte de mentores às EBTs normalmente será ofertado por AEs e IEs às suas EBTs. Esta oferta por estes dois tipos de agentes se deve ao fato de suas EBTs estarem em um ciclo inicial de desenvolvimento, necessitando de apoio de profissionais com conhecimento e com uma rede de relacionamentos consolidada no mercado.

Enquanto as AEs e IEs estão voltadas para o apoio à captação de investimentos privados, os PTecs, tanto portugueses quanto brasileiros, normalmente dão suporte à captação de recursos financeiros oferecidos por agentes públicos.
Este resultado contribui para a literatura, mostrando que o suporte financeiro obtido pelas EBTs com o auxílio dos ASIs varia de acordo com o tipo de ASI e com o seu nível de desenvolvimento. 


\section{Considerações Finais}

Este artigo apresenta uma análise detalhada do papel de AEs, IEs e PTecs no apoio ao desenvolvimento de EBTs. A este respeito, foi possível identificar como estes agentes dão apoio às EBTs em aspectos relativos ao espaço físico e serviços básicos, às práticas gerenciais, à aculturação e à construção de redes de relações sociais, aprofundando os conhecimentos vigentes sobre estes três tipos de agentes (ex.: Bollingtoft, 2012; Carayannis \& Zedtwitz, 2005; Paradkar et al., 2015; Pauwels et al., 2016; Vanderstraeten \& Matthyssens, 2012).

Com efeito, a resposta para a questão de pesquisa apresentada na introdução deste artigo foi racionalizada e discutida na seção anterior.

Como contribuição, este artigo apresenta uma visão abrangente e integradora do papel dos ASIs em diversos aspectos do desenvolvimento de EBTs, que vai além das visões até então apresentadas na literatura, que normalmente se mostram concentradas em aspectos específicos relativos aos ASIs. São também delineadas semelhanças e diferenças entre os três tipos de ASIs pesquisados, no que diz respeito ao seu papel em apoiar EBTs, aprimorando a literatura. Da perspectiva teórica, foi possível identificar especificidades em cada tipo de ASI, no que tange ao seu apoio ao desenvolvimento de EBTs.

Quanto às suas implicações para a prática, este artigo pode servir como ponto de referência para novos empreendedores, no sentido de que estes entendam melhor os papéis e serviços que podem ser providos em cada tipo de ASI e possam elaborar uma lista de pontos (um checklist) de elementos relevantes para o desenvolvimento de suas empresas. Paralelamente, este artigo pode ser utilizado por gestores de ASIs como elemento de referência para a avaliação do seu estado atual, enquanto provedores de serviços para EBTs, assim como para o seu desenvolvimento futuro, no sentido de expandirem seus serviços e atenderem cada vez melhor estas empresas.

Embora este estudo possa apresentar uma visão abrangente do papel dos ASIs no desenvolvimento das EBTs, posto que seu escopo se encontra baseado nos quatro construtos mencionados ao longo do texto, ele é limitado conceitualmente por estes mesmos construtos.

Não obstante, como sugestão de estudos futuros que permitam entender o comportamento de toda a população de ASIs, sugere-se o uso de toda a estrutura de construtos ora apresentada para a elaboração de estudos com caráter quantitativo para aplicação em todo o território brasileiro e português, para então se ter um panorama do comportamento dos ASIs na totalidade destes dois países. Outra sugestão é o uso de abordagens epistemológicas alternativas para a construção de teoria, como a Grounded Theory, entre outras (ex.: Charmaz, 2014; Mello \& Cunha, 2010; Shah \& Corley, 2006; Shepherd \& Suddaby, 2016), no sentido de se entender melhor o papel dos ASIs, a partir de uma abordagem que procure identificar novos construtos, tendo como pressuposto fundamental o contato do pesquisador com a realidade vivida pelos ASIs e EBTs, prescindindo inicialmente de qualquer viés teórico, visando, com isso, possibilitar o surgimento de perspectivas teóricas alternativas para este assunto.

\section{Referências}

Alvarez, I., Marin, R., \& Fonfria, A. (2009). The role of networking in the competitiveness of firms. Technological Forecasting \& Social Change, 76(3), 410-421. http://dx.doi.org/10.1016/j.techfore.2008.10.002

Andrade, P. P., Jr. (2014). Modelo de avaliação e superação das dificuldades de empresas de base tecnológica em incubadoras. Produção, 24(4), 809-819. http://dx.doi.org/10.1590/S010365132013005000085 
Antolín-López, R., Céspedes-Lorente, J., García-de-Frutos, N., Martínez-del-Río, J., \& Pérez-Valls, M. (2015). Fostering product innovation: Differences between new ventures and established firms. Technovation, 41-42, 25-37. http://dx.doi.org/10.1016/j.technovation.2015.02.0027

Audretsch, D. B., Lehmann, E. E., Paleari, S., \& Vismara, S. (2016). Entrepreneurial finance and technology transfer. Journal of Technology Transfer, 41(1), 1-9. https://doi.org/10.1007/s10961014-9381-8

Barbalho, S. C. M., Amaral, D. C., Kerbinchler, T. S., Ricther, E. H., \& Torres, L. (2009). Rompendo obstáculos para a implantação de escritório de projetos em empresa de base tecnológica. Gestão \& Produção, 16(3), 435-449. http://dx.doi.org/10.1590/S0104-530X2009000300010

Bertoni, F., Massimo, G., \& Grilli, L. (2011). Venture capital financing and the growth of high-tech start-ups: Disentangling treatment from selection effects. Research Policy, 40(7), 1028-1043. http://dx.doi.org/10.1016/j.respol.2011.03.008

Bigliardi, B., Dormio, A. I., Nosella, A., \& Petroni, G. (2006). Assessing science parks 's performance: directions from selected Italian case studies. Technovation, 26(4), 489-505. http://dx.doi.org/10.1016/j.technovation.2005.01.002

Bollingtoft, A. (2012). The bottom-up business incubator: Leverage to networking and cooperation practices in a self-generated, entrepreneurial-enable environment. Technovation, 32(5), 304-315. http://dx.doi.org/10.1016/j.technovation.2011.11.005

Bruneel, J., Ratinho, T., Clarysse, B., \& Groen, A. (2012). The evolution of business incubators: Comparing demand and supply of business incubation services across different incubator generations. Technovation, 32(2), 110-121. http://dx.doi.org/10.1016/j.technovation.2011.11.003

Caiazza, R., Richardson, A., \& Audretsch, D. (2015). Knowledge effects on competitiveness: From firms to regional advantage. Journal of Technology Transfer, 40(6), 899-909. http://dx.doi.org/10.1007/s10961-015-9425-8

Campanella, F., Peruta, M. R. D., \& Giudice, M. D. (2014). Creating conditions for innovative performance of science parks in Europe. How manage the intellectual capital for converting knowledge into organizational action. Journal of Intellectual Capital, 15(4), 576-596. http://dx.doi.org/10.1108/JIC-07-2014-0085

Carayannis, E. G., \& Zedtwitz, M. (2005). Architecting gloCal (global-local), real-virtual incubator networks (G=RVINs) as catalysts and acclerators of entrepreneuship in transitioning and developing economies: Lessons learned and best practices from current development and business incubation practices. Technovation, 25(2), 95-110. http://dx.doi.org/10.1016/S01664972(03)00072-5

Cesi, F., \& Lubatti, D. (2012). Personal relationships and innovation diffusion in SME networks: A content analysis approach. Research Policy, 41(3), 565-579. http://dx.doi.org/10.1016/j.respol.2011.10.003

Chan, K. F., \& Lau, T. (2005). Assessing technology incubator programs in the science park: The good, the bad and the ugly. Technovation, 25(10), 1215-1228. http://dx.doi.org/10.1016/j.technovation.2004.03.010

Charmaz, K. (2014). Grounded theory in global perspective: Reviews by international researchers. Qualitative Inquiry, 20(9), 1074-1084. http://dx.doi.org/10.1177/1077800414545235

Colombo, M. G., Cumming, D. J., \& Vismara, S. (2016). Governmental venture capital for innovative young firms. Journal of Technology Transfer, 41(1), 10-24. http://dx.doi.org/10.1007/s10961$014-9380-9$ 
Colyvas, J., Crow, M., Gelijns, A., Mazzoleni, R., Nelson, R. R., Rosenberg, N., \& Sampat, B. N. (2002). How do university inventions get into practice? Management Science, 48(1), 61-72. http://dx.doi.org/10.1287/mnsc.48.1.61.14272

Coradi, A., Heinzen, M., \& Boutellier, R. (2015). A longitudinal study of workspace design for knowledge exploration and exploration in the research and development process. Creativity and Innovation Management, 24(1), 55-71. http://dx.doi.org/10.1111/caim.12099

Costa, L. B. C., \& Torkomian, A. L. V. (2008). Um estudo exploratório sobre um novo tipo de empreendimento: Os spin-offs acadêmico. Revista de Administração Contemporânea, 12(2), 395427. http://dx.doi.org/10.1590/S1415-65552008000200006

Dosi, G. (2006). Mudança técnica e transformação industrial: A teoria e uma aplicação à indústria dos semicondutores. Campinas: Editora Unicamp.

Einsenhardt, K. M. (1989). Building theories from case study research. The Academy of Management Review, 14(4), 532-550. http://dx.doi.org/10.5465/AMR.1989.4308385

Franco, M., \& Haase, H. (2015). University-industry cooperation: Researchers' motivations and interaction channels. Journal of Engineering and Technology Management, 36, 41-51. http://dx.doi.org/10.1016/j.jengtecman.2015.05.002

Freman, C., \& Soete, L. (2008). A economia da inovação industrial. Campinas: Editora Unicamp.

Gaino, A. A. P., \& Pamplona, J. B. (2014.) Abordagem teórica dos condicionantes da formação e consolidação dos parques tecnológicos. Produção, 24(1), 177-187. http://dx.doi.org/10.1590/S0103-65132014000100014

Godoy, A. S. (1995). A pesquisa qualitativa e sua utilização em administração de empresas. Revista de Administração de Empresas, 35(4), 65-71. http://dx.doi.org/10.1590/S003475901995000400008

Godoy, A. S. (2010). Estudo de caso qualitativo. In C. K. Godoi, R. B. Mello, \& A. Silva (Orgs.), Pesquisa qualitativa em estudos organizacionais: Paradigmas, estratégias e métodos (pp.115143). São Paulo: Editora Saraiva.

Hansson, F., Husted, K., \& Vestergaard, J. (2005). Second generation science parks: From structural holes jockeys to social capital catalystis of the knowledge society. Technovation, 25(9), 10391049. http://dx.doi.org/10.1016/j.technovation.2004.03.003

Heuven, J., \& Groen, A. (2012). The role of social networks in financing technology-based ventures: An empirical exploration. Venture Capital, 14(2/3), 131-149. http://dx.doi.org/10.1080/13691066.2012.659473

Hueske, A., Endrikat, J., \& Guenther, E. (2015). External environment, the innovating organization, and its individuals: A multilevel model for identifying innovation barriers accounting for social uncertainties. Journal of Engineering and Technology Management, 35, 45-70. http://dx.doi.org/10.1016/j.jengtecman.2014.10.001

Hughes, M., Ireland, R. E., \& Morgan, R. E. (2007). Stimulating dynamic value: Social capital and business incubation as a pathway to competitive success. Long Range Planning, 40(2), 154-177. http://dx.doi.org/10.1016/j.lrp.2007.03.008

Iacono, A., Almeida, C. A. S. de, \& Nagano, M. S. (2011). Interação e cooperação de empresas incubadas de base tecnológica: Uma análise diante do novo paradigma da inovação. Revista de Administração Pública, 45(5), 1485-1516. http://dx.doi.org/10.1590/S003476122011000500011 
Jaskiewicz, P., Heinrichs, K., Rau, S. B., \& Reay, T. (2016). To be or not to be: How family firms manage family and comercial logics in sucession. Entrepreneurship, Theory and Practice, 40(4), 781-813. http://dx.doi.org/10.1111/etap.12146

Jennings, J. E., Edwards, T., Jennings, P. D., \& Delbridge, R. (2015). Emotional arousal and entrepreneurial outcomes: Combining qualitative methods to elaborate theory. Journal of Business Venturing, 30(1), 113-130. http://dx.doi.org/10.1016/j.jbusvent.2014.06.005

Lindstrom, G., \& Olofsson, C. (2001). Early stage financing of NTBF-s: An analysis of contributions from support actors. Venture Capital, 3(2), 151-168. http://dx.doi.org/10.1080/13691060110042754

Liu, F., Simon, D. F., Sun, Y., \& Cao, C. (2011). China's innovation policies: Evolution, institutional structure, and trajectory. Research Policy, 40(7), 917-931. http://dx.doi.org/10.1016/j.respol.2011.05.005

Lockett, A., \& Wright, M. (2005). Resources, capabilities, risk capital and the creation of university spin-out companies. Research Policy, 34(7), 1043-1057. http://dx.doi.org/10.1016/j.respol.2005.05.006

Malek, K., Maine, E., \& McCarthy, I. P. (2014). A typology of clean technology commercialization accelerators. Journal of Engineering and Technology Management, 32, 26-39. http://dx.doi.org/10.1016/j.jengtecman.2013.10.006

McAdam, M., \& McAdam, R. (2008). High tech start-ups in university science parks incubators: The relationship between the star-up's lifecycle progression and use of the incubator's resources. Technovation, 28(5), 277-290. http://dx.doi.org/10.1016/j.technovation.2007.07.012

Mello, R. B., \& Cunha, C. J. C. A. (2010). Grounded theory. In C. K. Godoi, R. B. Mello, \& A. Silva (Orgs.), Pesquisa qualitativa em estudos organizacionais: Paradigmas, estratégias e métodos (pp. 241-258). São Paulo: Editora Saraiva.

Mian, S., Lamine, W., \& Fayolle, A. (2016). Technology business incubation: On overview of the state of knowledge. Technovation, 50-51, 1-12. http://dx.doi.org/10.1016/j.technovation.2016.02.005

Mintzberg, H., Lampel, J., Quinn, J. B., \& Goshal, S. (2006). O processo de estratégia: Conceitos contextos e casos selecionados. Porto Alegre: Bookman.

Nagano, M. S., Stefanovitz, J. P., \& Vick, T. E. (2014). Innovation management processes, their internal organizational elements and contextual factors: An investigation in Brazil. Journal of Engineering and Technology Management, 33, 63-92. http://dx.doi.org/10.1016/j.jengtecman.2014.02.004

Ndonzuau, F. N., Pirnay, F., \& Surlemont, B. (2002). A stage model of academic spin-off creation. Technovation, 22(5), 281-289. http://dx.doi.org/10.1016/S0166-4972(01)00019-0

Nicolaou, N., \& Birley, S. (2003). Social networks in organizational emergence: The university spinout phenomenon. Management $\quad$ Science, $49(12), \quad 1702-1725$. http://dx.doi.org/10.1287/mnsc.49.12.1702.25116

Offord, M., \& Gill, R. (2016). Leadership between decks: A synthesis and development of engagement and resistance theories of leadership based on evidence from practice in Royal Navy warships. Leadership \& Organization Development Journal, 37(2), 289-304. http://dx.doi.org/10.1108/LODJ-07-2014-0119

Paradkar, A., Knight, J., \& Hansen, P. (2015). Innovation in start-ups: Ideas filling the void or ideas devoid of resources and capabilities? Technovation, 41-42, 1-10. http://dx.doi.org/10.1016/j.technovation.2015.03.004 
Pauwels, C., Clarysse, B., Wight, M., \& Hove, J. van (2016). Understanding a new generation incubation model: The accelerator. Technovation, 50-51, 13-26. http://dx.doi.org/10.1016/j.technovation.2015.09.003

Perussi, S., Filho, \& Escrivão, E., Filho (2012). Processo de criação de estratégia em pequenas empresas de base tecnológicas: Um modelo de fases evolutivas para o setor médico-odontológico. Gestão \& Produção, 19(1), 173-188. http://dx.doi.org/10.1590/S0104-530X2012000100012

Ratinho, T., \& Henriques, E. (2010). The role of science parks and business incubators in covering countries: Evidence from Portugal. Technovation, 30(4), 278-290. http://dx.doi.org/10.1016/j.technovation.2009.09.002

Raupp, F. M., \& Beuren, I. M. (2011). Perfil do suporte oferecido pelas incubadoras brasileiras às empresas incubadas. Revista Eletrônica de Administração, 17(2), 330-359. Recuperado de http://www.scielo.br/pdf/read/v17n2/02.pdf. 23112011000200002

Ribeiro, F. F., Oliveira, M. M., Jr., \& Borini, F. M. (2012). Internacionalização acelerada de empresas de base tecnológica: $\mathrm{O}$ caso das Born Globals brasileiras. Revista de Administração Contemporânea, 16(6), 866-888. Recuperado de http://www.scielo.br/pdf/rac/v16n6/a07v16n6.pdf. $\quad$ http://dx.doi.org/10.1590/S141565552012000600007

Robbins, P., \& Gorman, C. O. (2015). Innovating the innovation process: An organizational experiment in global pharma pursuing radical innovation. $R \& D$ Management, 45(1), 76-93. http://dx.doi.org/10.1111/radm.12054

Rubin, T. H., Aas, T. H., \& Stead, A. (2015). Knowledege flow in technological business incubators: Evidence from Australia and Israel. Technovation, 41-42, 11-24. http://dx.doi.org/10.1016/j.technovation.2015.03.002

Saunila, M., \& Ukko, J. (2014). Intangible aspects of innovation capability in SMEs: Impacts of size and industry. Journal of Engineering and Technology Management, 33, 32-46. http://dx.doi.org/10.1016/j.jengtecman.2014.02.002

Schwartz, M., \& Hornych, C. (2008). Specialization as strategy for business incubadors: An assessement of the central German multimedia center. Technovation, 28(7), 436-449. http://dx.doi.org/10.1016/j.technovation.2008.02.003

Scillitoe, J. L., \& Chakrabarti, A. K. (2010). The role of incubator interactions in assisting new ventures. Technovation, 30(3), 155-167. http://dx.doi.org/10.1016/j.technovation.2009.12.002

Shah, S. K., \& Corley, K. G. (2006). Buiding better theory by bridging the quantitative - qualitative divide. Journal of Management Studies, 43(8), 1821-1835. http://dx.doi.org/10.1111/j.14676486.2006.00662.x

Shepherd, D., \& Suddaby, R. (2016). Theory building: A review and integration. Journal of Management, 43(1), 1-28. http://dx.doi.org/10.1177/0149206316647102

Silva, J. M. da, Silva, C. E. S. da, Batista, G. D. M., \& Bittencourt, M. P. (2012). Impacto das funções desempenhadas pelos gerentes nos resultados da incubadora: Survey realizada na rede mineira de inovação. Produção, 22(4), 718-733. http://dx.doi.org/10.1590/S0103-65132011005000067

Silva, M. A. O., Gomes, L. F. A., \& Correia, M. F. (2009). Cultura e orientação empreendedora: Uma pesquisa comparativa entre empreendedores em incubadoras no Brasil e em Portugal. Revista de Administração Contemporânea, 13(1), 57-71. Recuperado de http://www.scielo.br/pdf/rac/v13n1/a05v13n1.pdf. $\quad$ http://dx.doi.org/10.1590/S141565552009000100005 
Silva, S. E., \& Reis, L. P. (2015). O processo de estruturação de recursos no contexto de uma empresa de base tecnológica de origem acadêmica (EBTA). Revista de Administração e Inovação, 12(2), 153-179. http://dx.doi.org/10.11606/rai.v12i2.100337

Soetanto, D. P., \& Jack, S. L. (2013). Business incubators and the networks of technology-based firms. Journal of Technology Transfer, 38(4), 432-453. http://dx.doi.org/10.1007/s10961-011-9237-4

Sofouli, E., \& Vonortas, N. (2007). S\&T parks and business incubators in middle-sized countries: The case of Greece. Journal of Technology Transfer, 32(5), 525-544. http://dx.doi.org/10.1007/s10961-005-6031-1

Suddaby, R., \& Bruton, G. D., \& Si, S. X. (2015). Entrepreneurship through a qualitative lens: Insights on the construction and/or discovery of entrepreneurial opportunity. Journal of Business Venturing, 30(1), 1-10. http://dx.doi.org/10.1016/j.jbusvent.2014.09.003

Tenzer, H., \& Pudelko, M. (2015). Leading across language barriers: language-induced emotions in multinational teams. The Leadership Quarterly, 26(4), 606-625. http://dx.doi.org/10.1016/j.leaqua.2015.05.006

Vandenbroucke, E., Knockaert, M., \& Ucbasaran, D. (2016). Outside board human capital and early stage high-tech firm performance. Entrepreneurship Theory and Practice, 40(4), 759-779. http://dx.doi.org/10.1111/etap.12141

Vanderstraeten, J., \& Mathyssens, P. (2012). Service-based differentiation strategies for business incubators: Exploring external and internal alignment. Technovation, 32(12), 656-670. http://dx.doi.org/10.1016/j.technovation.2012.09.002

Vial, I. D., \& Montoro-Sanches, A. (2016). How knowledge links with universities may foster innovation: The case of a science park. Technovation, 50-51, 41-52. http://dx.doi.org/10.1016/j.technovation.2015.09.001

Villareal, O., \& Calvo, N. (2015). From the triple helix model to the global open innovation model: A case study based on international cooperation for innovation in Dominican Republic. Journal of $\begin{array}{lllll}\text { Engineering } \quad \text { and } & \text { Technology }\end{array}$ http://dx.doi.org/10.1016/j.jengtecman.2014.10.002

Yin, R. K. (1994). Case study research: Design and methods (2nd ed). Thousands Oaks: Sage Publications.

\section{Dados dos Autores}

\section{Sergio Evangelista Silva}

Rua 37, 115, 35400-000, Loanda, João Monlevade, MG, Brasil. E-mail: sergio@ deenp.ufop.br; silvaevangelista@gmail.com. https://orcid.org/0000-0002-3507-518X

Carlos Alberto Gonçalves

Av. Pres. Antônio Carlos, 6627, 31270-901, Pampulha, Belo Horizonte, MG, Brasil. E-mail: carlos@ face.ufmg.br

Joaquim Ramos Silva

Rua Miguel Lúpi, 20, 1200-781, Lisboa, Portugal. E-mail: jrsilva@ iseg.ulisboa.pt

Ana Isabel Ortega Venâncio

Rua Miguel Lúpi, 20, 1200-781, Lisboa, Portugal. E-mail: avenancio@ iseg.ulisboa.pt. https://orcid.org/0000-0001-8391-7575 


\section{APÊNDICE}

\section{Roteiro de Pesquisa}

\section{Questões sobre o entrevistado:}

Nome:

Formação:

Função:

Idade:

\section{Questões Relativas ao ASI:}

1. Espaço físico

1.1. Qual é a área destinada ao ASI?

1.2. Como as EBTs estão alocadas no presente espaço físico? Explique.

1.3. É permitida a associação apenas a empresas localizadas no agente? Explique.

1.4. Como é feita a cessão do espaço, sob a perspectiva econômica? Como as firmas retribuem o uso do espaço (aluguel, cessão pública, etc.)? Explique.

1.5. Que tipos de serviços estão presentes no espaço físico?

1.6. Como foi concebido o espaço físico deste ASI?

2. Serviços

2.1. Fale sobre os serviços de infraestrutura oferecidos às EBTs.

2.2. Que serviços adicionais são oferecidos às EBTs?

2.3. Fale sobre os mecanismos de assessoria, suporte e controle das EBTs hospedadas:

3. Aculturação

3.1. O ASI oferece algum tipo de treinamento gerencial? Se sim, que ferramentas são vistas?

3.2. O ASI oferece suporte de consultores internos?

3.3. O ASI oferece suporte de tutores?

4. Sistema social

4.1. Como é a interação com outras EBTs?

4.2. O ASI promove a interação interna? Como?

4.3. Como o ASI interage com as EBTs que hospeda?

4.4. O ASI promove a interação com agentes externos? 